\title{
Design of a Control System of Single-Head Pneumatic Hinging Machine Based on STC90C58
}

\author{
Xie $\mathrm{Yu}$ \\ School of Mechanical Engineering, \\ Wenzhou Vocational \& Technical College, Wenzhou 325035, \\ Zhejiang,China
}

\begin{abstract}
The spectacle frame and earpiece of plate glasses frame are connected by a hinge. The hinge is welded into the plastic spectacle frame or temple tip by using microcomputer control, but the hinging equipment is very expensive. So STC90C58 is chosen as the control core. We have designed a low-cost control system of single-head pneumatic hinging machine for glasses with large LCD screen as the work status displayer, which has the characteristics like simple operation, high anti-interference ability, scalability, versatility and reliability, precise processing timing and large drive current, etc.
\end{abstract}

Keywords-STC90C58; plate glasses; hinging machine; control system; STC90C58

CLC No. TM571

[Documentary identification code] A

\section{INTRODUCTION}

The spectacle frame and earpiece are connected by a hinge. The hinge between the spectacle frame and earpiece of a plate frame is generally welded into the plastic spectacle frame or temple tip under instantaneous high temperature, which has high requirements on the heating temperature and the accuracy of welding time.

Some large glasses manufacturers usually import microcomputer control devices for hinging glasses, but the equipment is expensive. Small businesses often purchase manual or semi-automatic hinge machine to weld hinges.
Semi-automatic hinge machine commonly uses intermediate relay, time relay and mechanical counter for the implementation of control, the advantage of which is low-cost. But the disadvantages are low accuracy in the time set, low degree of automation, the single function, complex wiring, inconvenience in performance extending, lack of human-computer interaction and poor stability.

\section{The Function AnAlysis Of The CONTROL System OF HINGE MACHINE FOR GLASSES}

The pneumatic single-head hinge machine for glasses consists of the engine seat, the fixture clamping the hinge, the pneumatic feed mechanism promoting the fixture to move down and the adjustable base of the spectacle frame and earpiece of glasses. The pneumatic clamps mechanism includes cylinder, piston cylinder, insulating plate and copper mouth. The pneumatic feed mechanism is made up of a cylinder, piston cylinder, bracket, bearing, etc. The adjustable base consists of dovetail slide blocks which can horizontally move from the front and back, left and right.

In order to accurately finish the glasses hinging process, we need to complete the following working procedures: loosing the clamp and hinge, clamping the hinge, heating, lowering the platform, hinging, blowing and cooling, lifting the platform, counting, etc, as shown in Figure 1.

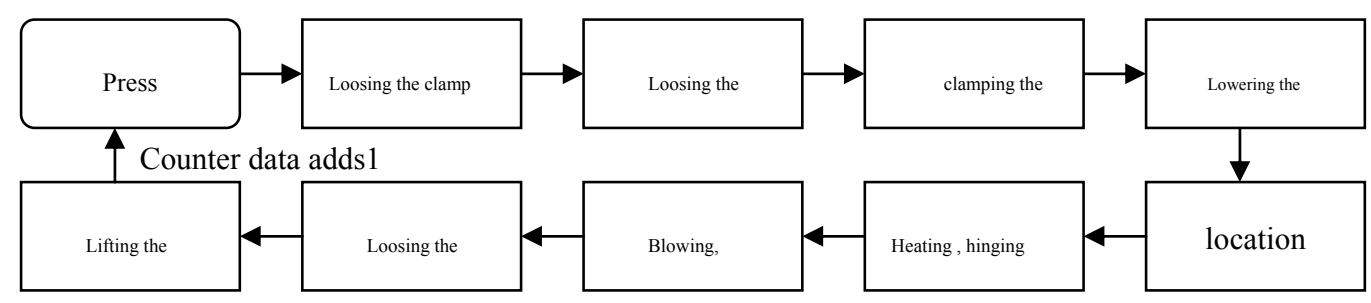

Figure 1 Glasses hinging machine workflow

Wherein the loosing and clamping of the clamp, the rising and falling of the cylinder and blowing and cooling are all pumped with $0.5 \mathrm{MPA}$ gas source provided by the pump and achieve the on-off control of corresponding gas circuit via a solenoid valve.

According to the different size and shape of the sheet material and hinge, the heating temperature is divided into many files with their separate heating circuits. The working times of processes as heating, a staple hinge and blowing and cooling also vary from 0.1 to 10 seconds.
The hinging machine could achieve manual single-process debugging, process time parameter adjustment, automatically hinging, counting and the corresponding protection function; the switch like the establishment of start, stop, manually loosing the clamp and manually lifting and declining the cylinder, etc. 


\section{The Design of CONTROL System OF THE GLASSES HINGING MACHINE}

Based on the analysis of the control functions of glasses hinging machine, the hinging machine control system needs to collect input switch signals like start, stop, the manually release of fixture, the manual rise and decline of cylinder, 0 to 9 keys and some information of the function keys to

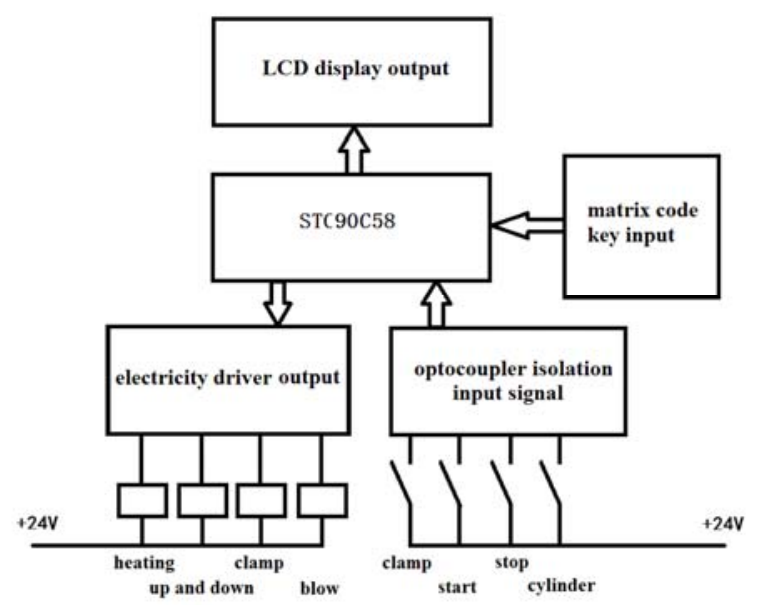

Figure 2 the system block diagram of glasses hinging machine.

\section{A. The design of hardware circuit}

\section{1) The circuit design of the control centre link}

The interface circuit of STC90C58 chip is shown in Figure 3, and the pin functions setting are shown in Table 1.

Wherein P0.0-P0.7 is acted as the interface of LCD data port / button matrix code and P1.0-P1.2 is acted as the selection port of " 20 " key matrix code.

\section{B. The circuit design of the control signal of switch quantity.}

After the operator give out the corresponding instruction: clamping, lifting cylinder, declining the hinge, start and stop according to a different process, the corresponding contact terminals are closed. For improving the anti-jamming capability, the signal is sent to the corresponding input end of single chip microcontroller after P521 optocoupler isolation. Considering the scalability of the machine, the 6-circuit optocoupler isolation circuit is set, its schematic diagram of single-way electrical shown in Figure 4. control heating, clamp, the rise and decline of cylinder and blowing and cooling, etc. and through large-screen LCD display the work state, the number of processing information, etc. A STC $90 \mathrm{C} 58$ chip is chosen as the core design motherboard circuit of the control system of pneumatic single-head glasses hinging, the system block diagram shown of which is shown in Figure 2.

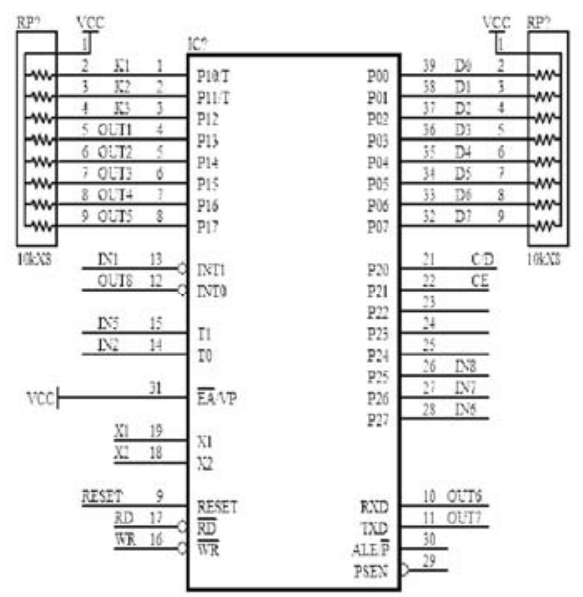

Figure 3 the interface circuit of STC $90 \mathrm{C} 58$ chip

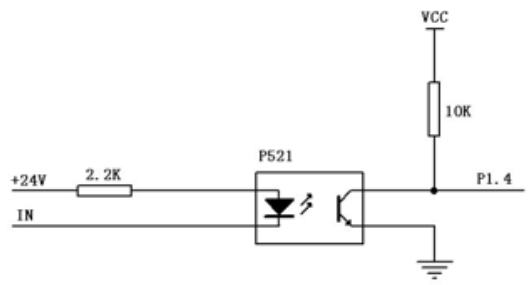

Figure 4 The collection of hinging machine input signal

\section{The circuit design of information coding input.}

To enhance the human-computer interaction capabilities of glasses hinging machine system with pneumatic single-head, and for the convenience of parameter setting and some functional command input, we provide functions like two operation mode (manual / automatic)to be selected, the setting and confirmation of the processing time ( 0.1 to 99.9 seconds), the counting data reset and the cursor's four-direction shift. To reduce the input points, the input keyboard of 20-dot matrix code is set by encoding circuit to input and confirm information, the electrical schematic diagram of input keyboard setting of 20-dot matrix code shown in Figure 5, the setting of hinging machine panelset shown in Figure 6. 


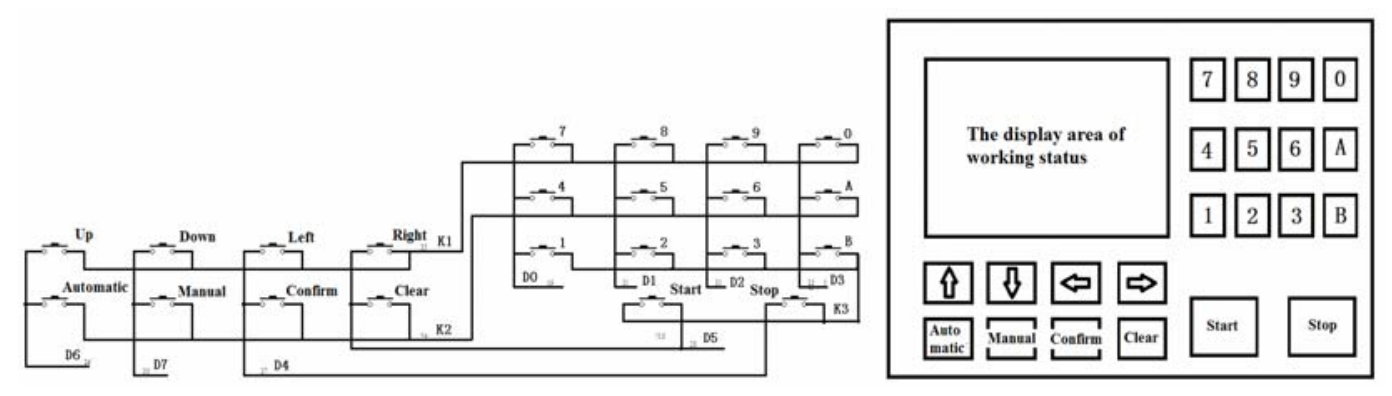

Figure 5 The electrical schematic diagram of input kevboard setting of 20-dot matrix code.

Figure 6 The setting of hinging machine panelset.

Table 1 the setting table of STC90C58 pin functions

\begin{tabular}{c|c|c|c|c|c|c|c}
\hline \multicolumn{2}{c|}{ pin } & number & function & \multicolumn{2}{|c|}{ pin } & number & function \\
\hline $1 \sim 3$ & P1.0 P1.2 & K1 $\sim$ K3 & $\begin{array}{c}\text { Matrix code selection end } \\
\text { of keys }\end{array}$ & 16 & $\overline{W R}$ & & LCD writing signal \\
\hline $4 \sim 8$ & P1.3 P1.7 & OUT1 OUT5 & Output point & 17 & $\overline{R D}$ & & LCD reading signal \\
\hline 9 & RESET & RESET & reset & 18 & $\mathrm{X} 2$ & & crystal oscillator input \\
\hline 10 & RXD & OUT6 & Output point & 21 & P2.0 & C/D & LCD data/Command selection end \\
\hline 11 & TXD & OUT7 & Output point & 22 & P2.1 & CE & LCD enable signal \\
\hline 12 & TXT0 & OUT8 & Output point & 26 & P2.5 & IN8 & input point \\
\hline 13 & TXT1 & IN1 & input point & 27 & P2.6 & IN7 & input point \\
\hline 14 & T0 & IN2 & input point & 28 & P2.7 & IN6 & input point \\
\hline & & & input point & $32--39$ & P0.0-P0.7 & D0-D7 & LCD data link/key matrix code \\
\hline
\end{tabular}

\section{LCD display interface design.}

To better display the working condition of hinging machine, a large LCD screen is chosen as a working status displayer. The heating time, release time, blowing time, return time, clamping, manual / automatic status and process counting and other information display area are designed on the LCD screen. And set the relevant parameters through the keyboard, the correspondent image area settings shown in Figure 7 and the LCD pin function settings shown in Figure 8.

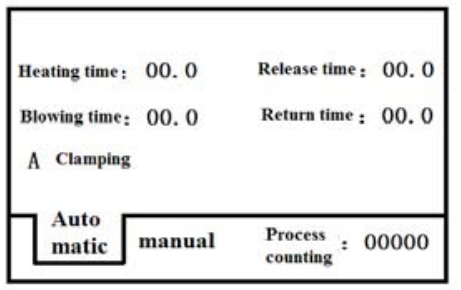

Figure 7 the display area setting of the working status of hinging machine.

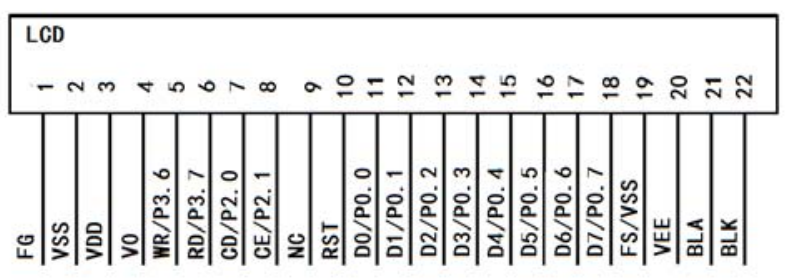

Figure 8 the LCD pin function settings 


\section{E. The design of driver output circuit.}

The pneumatic single-head hinging machine for glasses is required to achieve functions like control heating of hinging machine, cylinder's shift up and down, the clamping / releasing of the workpiece, the blowing and cooling after welding in its workflow. The components need high current to keep running, while the output signal current of single chip microcomputer is low. So to

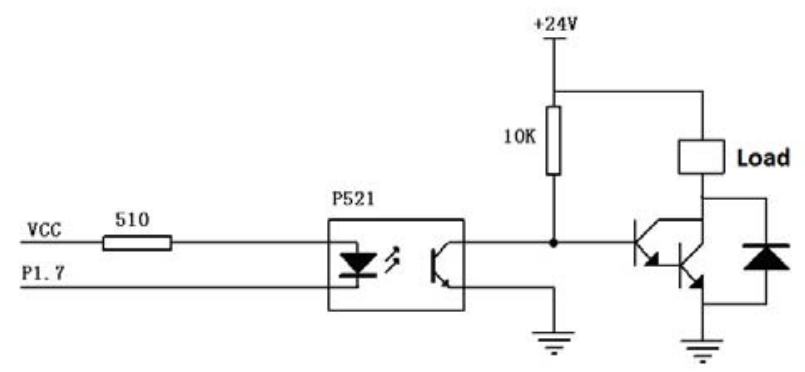

Figure 9 the amplifying circuit of the output current of hinging machine

\section{F. The software design of the pneumatic single-head hinging machine of glasses.}

According to the requirements of the workflow and parameter settings of hinging machine, the flow chart of the hinging machine control is designed, shown in Figure 10.

After the hinging machine power on, system initialization and displaying manufacturer and machine information, after 2 seconds, the working interface is shown in Figure 6. The default state is automatic operation.

\section{G. Parameter adjustment.}

On the work interface, press the "OK" button, entering the parameter setting interface, where the cursor is flashing in heating time: 00.0. Move the cursor to the position of time adjusting through the four cursor movement keys and, press the number keys directly to change the data.

\section{H. Manual debugging.}

On the work interface, press the "Manual" button to enter manufacturer debugging interface. Press the number keys $1,2,3$, and 4 to respectively, individually control the release of clamp, heating, the up and down shift and blowing action of cylinder and some related process debugging.

\section{Automatic operation.}

On the working interface, directly press the "Start" button, enter a complete workflow : fixture loosen $\rightarrow$ the hinge released $\rightarrow$ fixture clamped $\rightarrow$ cylinder declining $\rightarrow$ heating $\rightarrow$ blowing and cooling $\rightarrow$ fixture loosen $\rightarrow$ cylinder rising. Once a complete process is completed, the guarantee the stability of working status, in addition to utilizing the optocoupler P521 for isolation process between the output and load of the single chip microcontroller, the current amplification link needs to be enhanced so that low current signal could control the high current load. Also considering the scalability of machine, 9-circuit current amplifier circuit is set, with its single-channel electrical schematic diagram shown in Figure 9.

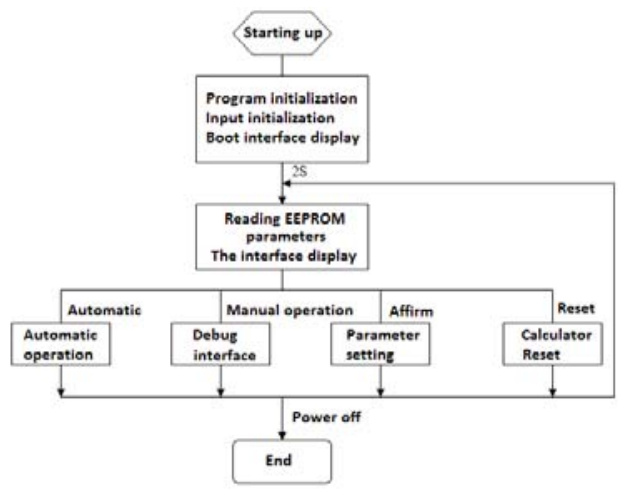

Figure 10 the flow chart of the hinging machine control

counter data plus 1. During the work, once the stop button is pressed, immediately ends and returns to the start.

\section{J. Reset the counter.}

On the work interface, press the "Clear" button to clear the data recorded by the counter inside.

\section{SUMMARY}

Considering the cheap but high-quality single-head hinging machine developed by small-scale Wenzhou glasses factory, we use SCM as the control core. The cost of each piece of equipment increase by 400 yuan and the factory price correspondingly increase by 600 yuan. The processing accuracy is improved and so is the economic efficiency of enterprises. The simpler operation makes it a great choice for enterprises.

On this basis, we will further increase technology investment and strengthen the control functions and display functions with human-computer interaction to generate greater economic benefits with low-cost investment.

\section{ACKNOWLEDGEMENTS}

This article was the final paper of research projects of Wenzhou Second Phase Of Science And Technology Plan Project In 2012 (G20120017)

Introduction of author: Xie Yu, male (1973-), born in Ruian, Lecturer in Department of Mechanical Engineering, Wenzhou Vocational \& Technical College, graduated from Industry Automation Major of Electrical Engineering Department of HeFei University of Technology in 1997 , Bachelor of Engineering, Master of Control Engineering, 
Wuhan University of Technology in 2008, currently engaged in research and teaching work on mechanical and electrical technology application.

Address: Department of Mechanical Engineering, Wenzhou Vocational \& Technical College in Wenzhou Chashan Higher Education Zone.

Tel: 13867705757

E-mail:189153298@qq.com

\section{REFERENCES}

[1] Zhang Qianjing, Chen Xiangtao. A stepper motor drive technology based on SCM andCPLD[J]. Microcomputer Applications, 2008, 29 (3): 71-74

[2] Lin Pingyong. Electrical and Electronic Technology, Beijing: Higher Education published social, 2008. 1: 171-173.

[3] Wang Feng, Zhang Lin. Electrical and electronic technology and application projects tutorials. Tianjin: Nankai University Press, 2010.8: 132-181.

[4] Ning Fan, the editor. 51 MCU-based tutorial. Beijing University of Aeronautics and Astronautics Press, 2008.

[5] Liu Song, the editor. SCM technology and applications. Machinery Industry Press, 2011. 